\title{
Nonviral genome engineering of natural killer cells
}

\author{
Gabrielle M. Robbins ${ }^{1,2,3,4+} \mathbb{D}$, Minjing Wang ${ }^{1,2,3+}$, Emily J. Pomeroy ${ }^{1,2,3+}$ and Branden S. Moriarity ${ }^{1,2,3^{*+}}$
}

\begin{abstract}
Natural killer (NK) cells are cytotoxic lymphocytes of the innate immune system capable of immune surveillance. Given their ability to rapidly and effectively recognize and kill aberrant cells, especially transformed cells, NK cells represent a unique cell type to genetically engineer to improve its potential as a cell-based therapy. NK cells do not express a T cell receptor and thus do not contribute to graft-versus-host disease, nor do they induce T cell-driven cytokine storms, making them highly suited as an off-the-shelf cellular therapy. The clinical efficacy of NK cell-based therapies has been hindered by limited in vivo persistence and the immunosuppressive tumor microenvironment characteristic of many cancers. Enhancing NK cell resistance to tumor inhibitory signaling through genome engineering has the potential to improve NK cell persistence in the tumor microenvironment and restore cytotoxic functions. Alongside silencing NK cell inhibitory receptors, NK cell killing can be redirected by the integration of chimeric antigen receptors (CARs). However, NK cells are associated with technical and biological challenges not observed in T cells, typically resulting in low genome editing efficiencies. Viral vectors have achieved the greatest gene transfer efficiencies but carry concerns of random, insertional mutagenesis given the high viral titers necessary. As such, this review focuses on nonviral methods of gene transfer within the context of improving cancer immunotherapy using engineered NK cells.
\end{abstract}

Keywords: Nonviral, NK cells, Genome engineering, Immunotherapy, Cell-based therapy, Transposon, CRISPR/CaS, Nucleofection, Lipofection

\section{Introduction}

Cancer therapy has been revolutionized through the adoptive transfer of tumor-infiltrating lymphocytes (TILs) and genetically engineered T cells. However, clinical efficacy has been largely limited to blood cancers [1]. Natural killer (NK) cells are innate lymphocytes with cytotoxic and inflammatory effector functions unleashed in response to cancer and thus represent an additional cell type of interest for cancer immunotherapy.

\footnotetext{
*Correspondence: mori0164@umn.edu

${ }^{\dagger}$ Gabrielle M. Robbins and Minjing Wang contributed equally.

${ }^{\dagger}$ Emily J. Pomeroy and Branden S. Moriarity contributed equally.

'Department of Pediatrics, University of Minnesota, Minneapolis, MN 55455,

USA

${ }^{2}$ Masonic Cancer Center, University of Minnesota, Minneapolis, MN 55455,

USA

Full list of author information is available at the end of the article
}

Moreover, NK cells do not express a $\mathrm{T}$ cell receptor (TCR) and thus have not been associated with some of the most detrimental side effects associated with $\mathrm{T}$ cellbased therapies, such as graft-vs-host disease (GvHD) and cytokine release syndrome (CRS), making NK cells an attractive candidate for cancer immunotherapy [2] However, studies to date have shown minimal clinical efficacy with unmanipulated peripheral blood NK cells. Thus, improving NK cell function through genetic modification is of high interest, but primary NK cells display resistance to many gene editing methods that work well in $\mathrm{T}$ cells [3-5]. A current consideration for engineered $\mathrm{T}$ and NK cell therapy is the method of gene transfer. The vast majority of engineered lymphocytes used in the clinic are made using viral vectors to deliver genetic material $[1,6]$. However, high cost of production, long

(c) The Author(s). 2021 Open Access This article is licensed under a Creative Commons Attribution 4.0 International License, which permits use, sharing, adaptation, distribution and reproduction in any medium or format, as long as you give appropriate credit to the original author(s) and the source, provide a link to the Creative Commons licence, and indicate if changes were made. The images or other third party material in this article are included in the article's Creative Commons licence, unless indicated otherwise in a credit line to the material. If material is not included in the article's Creative Commons licence and your intended use is not permitted by statutory regulation or exceeds the permitted use, you will need to obtain permission directly from the copyright holder. To view a copy of this licence, visit http://creativecommons.org/licenses/by/4.0/. The Creative Commons Public Domain Dedication waiver (http://creativecommons.org/publicdomain/zero/1.0/) applies to the data made available in this article, unless otherwise stated in a credit line to the data. 
turnaround times, batch to batch variability, and safety concerns have motivated the field to search for nonviral gene delivery and alternative approaches. Here, we review recent advances in the genetic modification of NK cells, with a focus on nonviral strategies.

\section{NK cell intransigence to genetic modification}

Viral vectors including retrovirus and lentivirus have been used extensively to stably deliver genetic material to a broad range of cell types [7]. They have been especially useful in the cell therapy field for the generation of engineered $\mathrm{T}$ cell therapies and hematopoietic stem/progenitor cell (HSPC) transplant. In contrast to $\mathrm{T}$ cells and HSPCs, NK cells are notoriously difficult to transduce $[3,8]$. High expression of pattern recognition receptors (PRRs) activated in response to pathogen- and danger-associated molecular patterns (PAMPs and DAMPs) may in part explain this phenomenon $[9,10]$. The RNA genome of retroviruses and lentiviruses is known to activate PRRs including toll-like receptor 3 (TLR3), retinoic acid-inducible gene I (RIG-I), and melanoma differentiation-associated protein 5 (MDA-5) in NK cells [8]. The result is poor NK cell viability and low transduction rates, which hinder the efficacy of this approach for therapeutic use [11].

\section{NK cell transfection strategies}

Transfection is a powerful tool for the deliberate introduction of nucleic acids into cells and can be used to deliver genome editing reagents for gene knockout or delivery of exogenous transgenes. Nonviral methods of transfection most often result in rapid, although transient, transgene expression when compared to viral-based methods $[4,12]$. A major concern with the viral modification of NK cells is the risk for insertional mutagenesis given the high viral titers necessary for successful transduction. Conversely, nonviral transfection-based methods do not carry the aforementioned risk, making them more favorable from a safety perspective, especially with an ultimate goal of developing novel immunotherapies. The most common methods of gene transfer using transfection include lipofection and electroporation (Table 1).
With lipofection, nucleic acids or proteins are encapsulated in cationic liposomes, which fuse with the target cell membrane [13]. Once fused, these liposomes release the cargo directly into the cell. While lipofection of NK cells has historically been used more sparingly, there are new bodies of research utilizing liposome-mediated transfection strategies [13]. One of the earliest studies using lipofection described the transfer of a murine interleukin-2 (IL-2) expressing plasmid into primary NK cells using 1,2-dimyristyloxy-propyl-3-dimethyl-hydroxy ethyl ammonium bromide/dioleoyl phosphatidylethanolamine (DMRIE/DOPE) [14]. IL-2 promotes proliferation and enhances the cytotoxicity of NK cells, including the secretion of granzyme. Investigators found that melanoma xenograft tumors treated with transfected NK cells had significantly higher levels of granzyme A activity [14]. The transformed NK cell line NK-92 (derived from peripheral blood mononuclear cells (PBMCs) of a nonHodgkin's lymphoma patient) [15] was lipofected with stem cell factor (SCF) cDNA and found to have significantly greater proliferation and stronger cytotoxicity against a broad range of target tumors when compared to wild-type NK-92 cells [16]. Lipofectamine 2000 has been used to transfect primary NK cells with an activating chimeric antigen receptor (CAR) specific to human epidermal growth factor receptor 2 (HER-2), an oncogene frequently overexpressed in a number of solid tumors. With transfection efficiencies averaging at $60 \%$ across ten different donors, these HER-2-specific CARNK cells were selectively activated by HER-2-positive tumor cells and eradicated tumor cells in vivo [17]. Youness et al. identified miR-486-5p as a direct regulator of insulin-like growth factor-1 receptor (IGF-1R), which is a known modulator of hepatocellular carcinoma [18]. miR-486-5p was lipofected into primary NK cells, resulting in improved NK cell cytotoxicity through an increase in natural killer group 2D (NKG2D) and perforin expression. Regis et al. found that miR-27a-5p negatively regulates $\mathrm{CX}_{3} \mathrm{C}$ chemokine receptor $1\left(\mathrm{CX}_{3} \mathrm{CR} 1\right)$, which drives $\mathrm{NK}$ cells to peripheral tissues, including tumor sites [19]. Investigators utilized Lipofectamine 3000 to transfect primary NK cells with a miR-27a-50 inhibitor

Table 1 Comparison of nonviral delivery strategies in NK cells

\begin{tabular}{llll}
\hline $\begin{array}{l}\text { Delivery } \\
\text { method }\end{array}$ & Advantages & Disadvantages & Apparatus \\
\hline $\begin{array}{llll}\text { Electroporation } \\
\text { Nucleofection }\end{array}$ & $\begin{array}{l}\text { High efficiency } \\
\text { Less regulatory constraints } \\
\text { cGMP compliant electroporation } \\
\text { systems }\end{array}$ & $\begin{array}{l}\text { NK cells must be expanded and require cytokines } \\
\text { Cell viability dependent on cargo (e.g., RNA, DNA) }\end{array}$ & $\begin{array}{l}\text { Neon } \\
\text { Amaxa } \\
\text { Cargo size affects efficiency }\end{array}$ \\
DNA, RNA, or plasmids & & MaxCyte \\
Lipofection & Cost effective & Cell viability dependent on cargo (e.g., RNA, DNA) & Lipofectamine 2000, \\
& Readily available reagents & Limited studies & 3000 \\
& DNA, RNA, or plasmids & Requires optimized conditions of reagents and cell & \\
& medium & \\
\hline
\end{tabular}


and achieved transfection efficiencies of $\sim 30 \%$. Hargreaves et al. compared the transfection efficiency and cellular viability of primary NK cells using different transfection techniques. Both transfection efficiency and cell viability ranged greatly from $0 \%$ up to $75 \%$ [20]. Most recently, Lipofectamine 2000 and 3000 were compared to a novel transfection reagent method known as charge-altering releasable transporters (CARTs). While lipofectaminetransfected primary NK cells had GFP detection below $1 \%$, CART-transfected NK cells expressed green fluorescent protein (GFP) more efficiently $(\sim 10 \%)$ and showed improved viability with minimal changes to NK cell phenotype and function [21]. These lipofected cells showed viabilities ranging from 40 to $85 \%$.

Electroporation-based methods are one of the earliest strategies used for nucleic acid delivery in NK cells. Electroporation is a method based on the generation of electrical pulses to induce small, temporary pores in the cell membrane [13]. These pores allow for charged molecules, such as DNA, RNA, and proteins, to move into the cell. Typically, electroporation requires target cells to be dividing in an exponential growth phase in order for nucleic acids to have optimal access to the nucleus. Previous studies demonstrate that primary NK cells require cytokine stimulation and/or expansion using feeder cell lines to allow for sufficient transfection efficiencies and post-electroporation viability [13, 22]. Nucleofection-based methods were developed to allow for efficient gene transfer into the cell nucleus without relying on cell division for nucleic acid transfer into the nucleus. Nucleofection uses the physical methods of electroporation (induction of cell membrane pores) but uses a unique device, known as a Nucleofector, as well as a combination of optimized electrical parameters and cell type-specific reagents [23]. Together, this enables the transfer of molecules directly into the cells' nucleus, which improves transfection efficiency and faster expression [24]. Using the nucleofector system, transfection efficiencies and cellular viability of NK cells vary greatly. A comparison of multiple transfection methods showed nucleofected NK cells had a transfection efficiency of $60 \%$ and post-transfection viability ranging from 50 to $75 \%$ [20]. Trompeter et al. aimed to optimize nucleofection of both primary NK cells and the IL-2dependent NK cell line, NKL. By testing variations in cell number and DNA amount, investigators achieved transfection efficiencies around $50 \%$ and cell viability inversely correlated with transfection efficiency due to the toxicity of the DNA [25].

\section{Transfection of NK cells with in vitro transcribed (IVT) mRNA}

While nucleofection methods are still employed, many investigators found that moving away from DNA-based cargo improved viability (Table 2). Carlsten et al. electroporated primary NK cells with mRNA to introduce a high-affinity CD16 and chemokine receptor C-C motif chemokine receptor 7 (CCR7) [26]. Greater than 95\% expression was achieved and engineered cells showed substantial migration to chemokine, C-C motif chemokine ligand 19 (CCL19), as well as greater cytotoxicity against antibody-coated lymphoma cells. While electroporation-based methods have been utilized with high efficiencies, viral transduction remains heavily used due to its ability for stable gene transfer. A comparison of mRNA electroporation and lentiviral transduction of the NK-92 cell line showed significantly greater transfection efficiencies and cytotoxicity when compared to mRNA electroporated cells [27]. Interestingly, cord blood NK cells had higher efficiencies when transduced virally, suggesting relevant differences between the NK92 cell line and primary NK cells [27]. Li et al. found electroporation efficiencies greater than $80 \%$ when they introduced an mRNA encoding a CAR receptor against

Table 2 Comparison of NK cell engineering reagents

\begin{tabular}{|c|c|c|c|c|c|}
\hline Cargo & Efficiency & Viability & Advantages & Disadvantages & Therapeutic uses \\
\hline $\begin{array}{l}\text { Transient mRNA } \\
\text { DNA }\end{array}$ & Up to $99 \%$ & Poor to good & $\begin{array}{l}\text { Rapid expression } \\
\text { High efficiency }\end{array}$ & $\begin{array}{l}\text { Transient-no stable genomic } \\
\text { integration } \\
\text { Cell viability dependent on cargo } \\
\text { (e.g., RNA, DNA) }\end{array}$ & $\begin{array}{l}\text { Transient CAR mRNA } \\
\text { Knockout of genes that suppress } \\
\text { or inhibit NK cell function } \\
\text { Knock-in of activating receptors } \\
\text { or genes that promote NK cell } \\
\text { function }\end{array}$ \\
\hline Transposon & Up to $80 \%$ & Poor to good & $\begin{array}{l}\text { Cost effective } \\
\text { Large cargo capacity } \\
\text { Stable integration }\end{array}$ & $\begin{array}{l}\text { Potential insertional mutagenesis } \\
\text { Transposon must be delivered } \\
\text { as DNA }\end{array}$ & $\begin{array}{l}\text { Large cargo delivery (e.g., CAR in } \\
\text { combination with activating } \\
\text { receptors or cytokines) }\end{array}$ \\
\hline $\begin{array}{l}\text { Cas9 } \\
\text { Base editor } \\
\text { Prime editor }\end{array}$ & Up to $100 \%$ & Poor to excellent & $\begin{array}{l}\text { High precision } \\
\text { High efficiency } \\
\text { Large-scale insertion } \\
\text { or deletion }\end{array}$ & $\begin{array}{l}\text { Potential off target editing } \\
\text { Indels and translocations }\end{array}$ & $\begin{array}{l}\text { Knockout genes that suppress or } \\
\text { inhibit NK cell function } \\
\text { Knock-in of activating receptors } \\
\text { or genes that promote NK cell } \\
\text { function } \\
\text { Treating patients bearing disease } \\
\text { caused by a single base pair } \\
\text { mutation }\end{array}$ \\
\hline
\end{tabular}


CD19 into rested (unstimulated) and expanded primary NK cells [28]. Both rested and expanded cells transfected with a CD19-CAR showed enhanced cytotoxicity against CD19+ targets when compared to non-transfected cells. Similarly, NK-92 cells have also been transfected with mRNA to express a CD19-CAR, chemokine receptor CCR7, as well as other reporter genes such as enhanced GFP (eGFP), yellow fluorescent protein (YFP), and Azuride [12]. Both viability and transfection efficiencies achieved were between 50 and $60 \%$, with CD19-CARtransfected cells showing improved cytotoxicity against $\mathrm{CD} 19+$ cell lines during in vitro killing assays. Importantly, delivery of activating receptors with mRNA or plasmid DNA results in transient expression, so alternative approaches are necessary to achieve stable expression of transgenes.

\section{NK cell engineering with DNA transposons}

A common strategy for stable, nonviral gene delivery is the use of DNA transposons (Table 2). Transposons, also known as transposable elements, are naturally existing repetitive DNA sequences that are capable of mobilizing from one location to another in the genome [29]. When used for gene delivery, DNA transposons are generally a two-component system, with a transposon vector containing sequences to be mobilized flanked by terminal inverted repeats (TIRs) and a transposase enzyme that identifies the TIRs and excises and reintegrates the transposon [30]. Since their discovery 70 years ago by Barbara McClintock, the "cut and paste" mechanism of transposons has been used as a genetic tool for multiple purposes, ranging from genetic screening to insertional mutagenesis and transgenesis $[29,31]$. As we enter the era of gene therapy and personalized medicine, transposons have been used extensively as an alternative to the viral vector system for engineering human cells. Recently, transposon-engineered induced pluripotent stem cells (iPSCs) and $\mathrm{T}$ cells have been used in clinical trials, making the transposon system one of the most promising nonviral vector systems for stable gene transfer [30,32].

There are three major superfamilies of transposons commonly used for gene transfer in human cells, namely Tc1/mariner, piggyBac (PB), and hAT [29]. The most extensively studied transposon system for gene transfer, the Sleeping Beauty (SB) transposon system, belongs to the Tc1/mariner superfamily [29]. Since its discovery and molecular reconstruction from the genomes of salmonid fish [33], SB has undergone improvements through the generation of hyperactive mutants and transposon donor vector optimization [34, 35]. The most hyperactive variant developed so far, SB100X, is shown to have a 100-fold improvement of integration efficiency compared to the original SB transposase and is comparable to that of a viral vector system [35]. For example, in human CD34+ hematopoietic cells, SB100X is able to achieve up to $50 \%$ integration efficiency, compared to $40 \%$ with lentiviral vectors $[35,36]$. SB integration has a consensus target site TA. High-resolution genome-wide mapping showed SB integration favors introns, transcriptional units, upstream regulatory sequences, and microsatellite repeats [37, 38]. $\mathrm{PB}$ is another well-developed transposon system for stable gene transfer that was isolated from the cabbage looper moth $[29,39]$. The PB system shares a similar transposition mechanism with SB, and its efficiency has also been significantly improved by hyperactive mutant screening and transposon/transposase vector optimization [39, 40]. The current hyperactive PB (super-PB) also exhibits a comparable efficiency of transposition to viral vectors [39]. PB also exhibits a non-random integration pattern, but with slightly different preferences compared to SB. PB has a TTAA consensus target site and shows a tendency to integrate into introns, transcriptional start sites, and long terminal repeat elements [37, 38]. As a novel representative of the hAT family, the TcBuster transposon originated from the red flour beetle and is a rising star for gene transfer [41]. TcBuster was shown to be highly active in human cell lines, including HEK-293 and HeLa cells, and has a comparable transposition efficiency to PB and SB $[42,43]$. TcBuster has an integration pattern that is slightly different from $\mathrm{SB}$ and $\mathrm{PB}$. TcBuster favors a TA integration site, and as for genomic elements, it slightly favors transcription units, CpG islands, and transcription start sites [37, 38].

Engineering NK cells using transposable elements has gained fairly limited attention and even fewer publications thus far. Among these published studies, NK-92 is almost exclusively used [27, 44]. For instance, in collaboration with the Kaufman laboratory, we previously utilized NK-92 cells to screen mesothelin-specific CAR constructs to enhance NK cell activity [45]. We successfully expressed a panel of novel CAR architectures in NK-92 cells using PB or SB systems and demonstrated improved anti-tumor activities of CAR-expressing NK92 cells when co-cultured with mesothelin-expressing targets [45]. Another study focused on expressing a CD73-specific CAR in NK-92 cells [46]. Using the PB system, Matosevic's group delivered a CD73-CAR construct to NK-92 cells and showed potent killing ability against both solid tumor target cells and humanized CD73+ lung cancer patient-derived xenograft (PDX) models [46].

As an alternative to transfecting NK cells directly with transposon systems, transfecting iPSCs and differentiating them into NK cells allows for the circumvention of low transfection efficiency of plasmids in primary NK cells $[45,47]$. Using this approach, $\mathrm{Li}$ et al. were able to 
express optimized mesothelin-CAR constructs using a super-PB system in human iPSCs, followed by differentiation into NK cells [45]. Like NK-92, stable expression of mesothelin-CAR was achieved in iPSCs, and subsequent functional assays indicated enhanced tumor specificity and killing in iPSC-derived CAR-NK cells [45].

Our finite understanding of NK cells in general and their notorious aversion to transgene uptake and expression are likely to blame for the limited study of transposable elements in primary NK cells. Transposons, especially $\mathrm{SB}$ and $\mathrm{PB}$, have been used extensively in delivering CARs to $\mathrm{T}$ cells for treating both hematological and solid tumors, with targets including CD19 [48], CD33 [49], CD133 [50], epidermal growth factor receptor (EGFR) [51], mesothelin [52], and glypican 3 (GPC3) [53]. Among them, a CD19-CAR and a signaling lymphocyte activation molecule family member 7 (SLAMF7)-CAR, both generated using the SB system, have already entered phase I/II clinical trial for treating leukemia or lymphoma $[54,55]$ and multiple myeloma (MM) [56], respectively. These achievements in $\mathrm{T}$ cells offer both insight and a basis for applying the concept in primary NK cells. With a better understanding of NK isolation and expansion, as well as the improvements in SB technology through hyperactive mutant generation (SB11, SB100X) and transposon donor vector optimization (pT4) [34, 35], it is feasible to expand and apply transposon systems for engineering primary NK cells. To this end, our group performed proof-of-principle experiments delivering mRNA encoding SB11 or SB100X in combination with a minicircle DNA transposon encoding GFP to feeder cellexpanded primary human NK cells (Fig. 1). We show stable expression of GFP 21 days after electroporation, with $15 \%$ efficiency using SB100X, suggesting this is a viable approach for nonviral, transposon-based gene delivery to NK cells.

Because of their higher gene transfer efficiencies, viral vector systems, including lentivirus, adeno-associated virus (AAV), and retrovirus, are still the preferred vector system used in NK cell engineering. Nevertheless, transposon systems are shown to overcome many limitations of the viral vector systems. Larger cargo size is one of the most prominent features transposon systems have over viral vectors [57]. Transposons have at least 9 $\mathrm{kb}$ of cargo capacity, while the largest carrying capacity for viral vectors has an 8-kb size limitation [57]. Moreover, SB was shown to have significantly lower integration rates than that of almost all the common viral vectors, with avian sarcoma leukosis virus (ASLV) being the only exception and sharing a similar rate as SB [37]. And though $\mathrm{PB}$ demonstrated a slightly higher integration frequency than $\mathrm{SB}$, the rate is still lower than that of HIV-1 [38]. Together, this indicates a greater safety profile of transposon systems than that of integrating viral vector systems. Additionally, transposon systems are more cost-effective and easier to produce and purify [29]. With the recent improvements of gene transfer efficiencies in transposon systems, especially that of SB and $\mathrm{PB}$, the efficiencies are comparable to that of viral
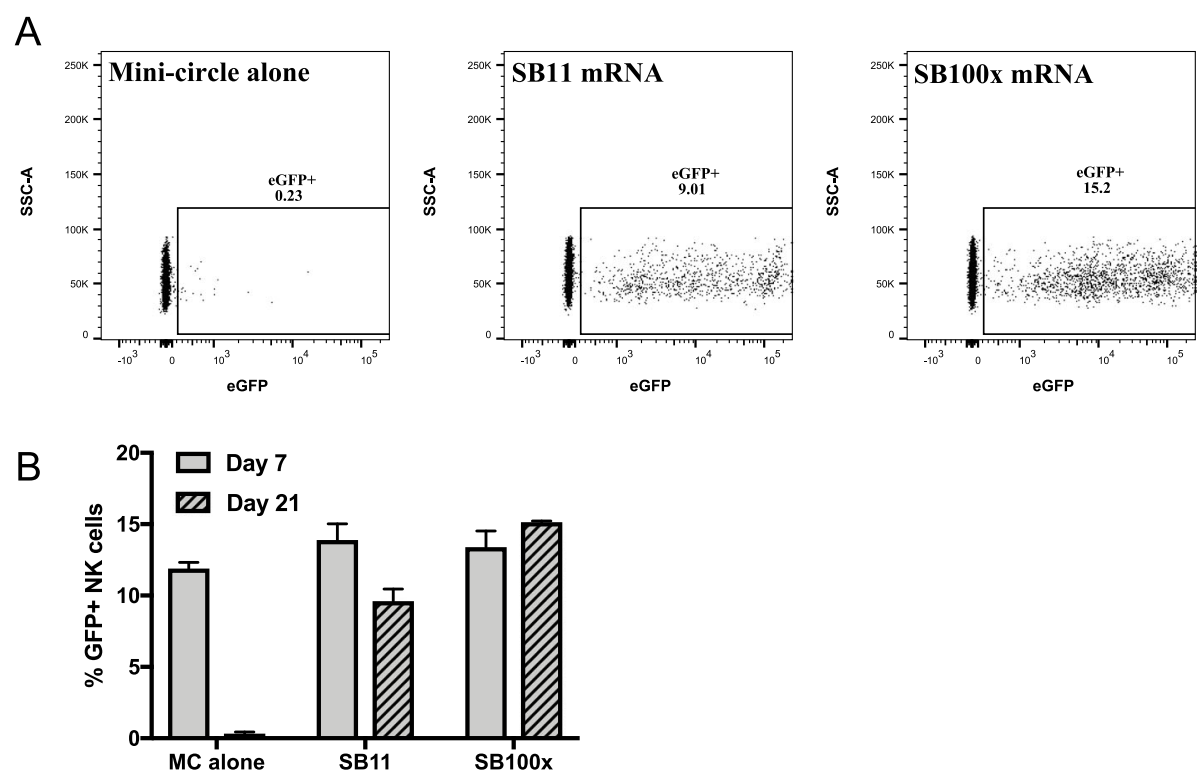

Fig. 1 SB mRNA and minicircle delivery of GFP to primary human NK cells. Primary human NK cells ( $n=2$ independent donors) were isolated from peripheral blood and expanded using membrane-bound interleukin-21 (mblL21)-expressing K562 feeder cells. After expansion, NK cells were electroporated with minicircle (MC) plasmid expressing eGFP alone or in combination with SB11- or SB100X-encoding mRNA. A Representative flow plots of eGFP expression 21 days after electroporation. B eGFP expression from 2 NK cell donors 7 and 21 days after electroporation 
vectors [35, 39], making transposon systems a very promising gene editing tool for NK engineering.

\section{NK cell engineering with Cas9, base editors, and prime editors}

The expression of an array of inhibitory receptors and checkpoint molecules that can be upregulated in the tumor microenvironment (TME) has made clustered regularly interspaced short palindromic repeats (CRIS PR)/CRISPR-associated protein 9 (Cas9)-based gene editing in NK cells a goal for scientists trying to augment NK cell anti-tumor efficacy [5] (Table 2). Early efforts to use CRISPR/Cas9 in primary NK cells used Cas9 expressed via a DNA plasmid or mRNA. This often resulted in low transfection efficiencies [58]. A method has recently been described to efficiently and reliably target genes for knockout with CRISPR/Cas9 in primary human NK cells using chemically modified guide RNAs and Cas9 mRNA [22]. Using this method, Pomeroy et al. targeted NK inhibitory genes (ADAM metallopeptidase domain 17) ADAM17 and (programmed cell death 1) $P D C D 1$ for knockout and developed a method for targeted integration using recombinant adeno-associated virus (rAAV) as a donor template for homology-directed repair (HDR). Gene knockout and knock-in efficiencies in this study reached $90 \%$ and $75 \%$, respectively, equivalent to reports of analogous approaches in primary $\mathrm{T}$ cells. Other groups have solved the problem of low transfection efficiency by delivering Cas9 and guide RNAs as ribonucleoprotein (RNP) complexes, as this approach has shown high editing rates in primary $\mathrm{T}$ cells [59]. Through the use of RNPs, Rautela et al. were able to achieve editing efficiencies of up to $75 \%$ across a number of genes in primary NK cells [60]. Others have used RNPs to efficiently knockout transforming growth factor beta receptor 2 (TGFBR2) in primary NK cells, as transforming growth factor beta (TGF $\beta$ ) is a major NK cell inhibitor [59]. Electroporation of cells with RNP complex to knockout TGFBR2 resulted in a $60 \%$ reduction in mRNA expression. Recently, Nguyen et al. developed methods to improve the efficiency of site-specific CRISPR/Cas9-based gene delivery, using Cas9-RNPs and DNA templates for HDR containing truncated Cas9 target sequences (tCTSs). The tCTSs associate with the Cas9-RNPs and are thus shuttled to the nucleus, which enhances HDR efficiency [61]. Using this approach, they achieved over $15 \%$ transgene delivery to NK cells.

Base editors (BEs) are another gene editing tool that takes advantage of the CRISPR/Cas system. BEs are composed of a catalytically inactive Cas 9 protein fused to a DNA deaminase domain [62]. Unlike Cas9 nuclease, this feature enables precise introduction of targeted single nucleotide changes without introductions of double-strand breaks (DSBs) or the need for a DNA donor molecule [63]. There are two types of base editors to date, adenine base editor (ABE) and cytosine base editor $(\mathrm{CBE})$, and collectively, they can achieve all possible transition mutations (A-> G for $\mathrm{ABE}$ and $\mathrm{C}->\mathrm{T}$ for $\mathrm{CBE}$ ) $[64,65]$. Due to its relatively recent development, applications of $\mathrm{BE}$ in the immunotherapy context are very limited. Studies using BE in T cells have just started emerging; however, applications in NK cells are still lacking. Last year, Webber and Lonetree et al. reported a multiplex knockout of $\mathrm{T}$ cell receptor alpha constant (TRAC), $\beta-2$ microglobulin $(B 2 M)$, and PDCD1 in CD19 CAR-T cells using both CBE base editors [66]. In this study, they achieved higher than $90 \%$ editing efficiency across all 3 target genes, at both DNA and protein levels [66]. Additionally, Zhang's group reported a downregulation of PD-1 expression in CAR-T cells using ABE [67]. By altering the coding sequencing of N74 in the $P D C D 1$ gene, they reduced asparagine $(\mathrm{N})$-linked glycosylation of PD-1 protein. This modification reduced its inhibitory effect on CAR-T cells. These studies serve as a proof of principle for using both $\mathrm{ABE}$ and $\mathrm{CBE}$ in $\mathrm{T}$ cells, and potentially NK cells, given the shared characteristics and functioning mechanisms between these two cell types.

Last year, Liu's team reported prime editor (PE), further expanding our ability to precisely engineer DNA without inducing DSBs or a need for DNA donor molecules $[62,68]$. PE uses a reverse transcriptase fused to dead Cas9 (dCas9) and prime editing guide RNA (pegRNA) containing a sequence to be introduced. PE is capable of introducing all possible transversion and transition mutations, as well as small insertions and deletions [68]. At its current stage, PE has only been tested in a very limited number of mammalian cells, including $293 \mathrm{~T}$ and $\mathrm{K} 562$, with up to $70 \%$ and 30\% editing efficiency, respectively [68]. However, sharing a similar mechanism as $\mathrm{BE}$, it is worth trying this technology in NK cells. If successful, this would greatly enhance our toolbox for editing NK cells using nonviral approaches.

\section{Summary}

NK cells have shown great promise as a cell-based therapy for cancer, but there is much work to be done to improve their anti-tumor efficacy. Recent advances in NK cell engineering, especially using nonviral methods, may unleash the therapeutic potential of NK cells as a cancer therapeutic. NK cell effector functions are regulated by an array of activating and inhibitory receptors. Cancer cells can evade NK cell detection by interacting with these receptors directly or by secreting immunosuppressive molecules. Therefore, genetic manipulation of NK activating or inhibitory receptors may augment antitumor activity. The most common nonviral delivery strategies for gene editing NK cells are lipofection and 
electroporation. While lipofection can be more costeffective than electroporation, it is clear that electroporation is the more efficient strategy and is well suited for clinical translation. Of particular interest are genome engineering reagents that confer stable gene delivery like transposons and gene editing tools that do not induce DSBs, such as BE and PE. As we continue to learn more about why NK cells resist transgene delivery, we can find new and creative ways to work around them and increase the efficiency of these techniques.

\section{Materials and methods}

\section{NK cell isolation and expansion}

PBMCs from de-identified healthy human donors were obtained by automated leukapheresis (Memorial Blood Centers, Minneapolis, MN, USA). CD56+CD- NK cells were isolated by negative selection using the EasySep Human NK Cell Enrichment Kit (STEMCELL Technologies). After isolation, NK cells were expanded by co-culture with irradiated (100 Gy) mbIL21- and 4-1BB ligand (41BBL)-expressing K562 feeder cells as described previously [21].

\section{Electroporation of expanded NK cells}

Expanded NK cells were pelleted and resuspended at $3 \times$ $10^{7}$ cells/mL in T buffer (Neon Transfection System Kit; Thermo Fisher Scientific). One microgram of MC plasmid DNA and $1 \mu \mathrm{g}$ SB11 or SB100X mRNA were added to $10 \mu \mathrm{L}\left(3 \times 10^{5}\right.$ cells $)$ on ice. This mixture was electroporated with the Neon Transfection System (Thermo Fisher Scientific) using two pulses of $1850 \mathrm{~V}$ and 10-ms pulse width. NK cells were re-expanded with feeder cells for 21 days.

\section{Flow cytometry}

The following antibodies were used: allophycocyanin (APC)-conjugated anti-CD56 (clone REA196; Miltenyi Biotec), phycoerythrin (PE)-conjugated anti-CD3 (clone SK7; BD Biosciences), and SYTOX Blue dead cell stain (Thermo Fisher). Flow cytometry assays were performed on LSR Fortessa flow cytometers (BD Biosciences), and data were analyzed using FlowJo version 10.4 software (FlowJo).

\footnotetext{
Abbreviations

41BBL: 4-1BB ligand; 5'UTR: 5' untranslated region; AAV: Adeno-associated virus; $A B E$ : Adenine base editor; ADAM17: ADAM metallopeptidase domain 17; APC: Allophycocyanin; ASLV: Avian sarcoma leukosis virus; B2M: B-2 microglobulin; BEs: Base editors; CAR: Chimeric antigen receptor; CARTs: Charge-altering releasable transporters; Cas9: CRISPR-associated protein 9; CBE: Cytosine base editor; CCL19: C-C motif chemokine ligand 19; CCR7: C-C motif chemokine receptor 7; CDNA: Complementary DNA; CRIS PR: Clustered regularly interspaced short palindromic repeats; CRS: Cytokine release syndrome; $\mathrm{CX}_{3} \mathrm{CR} 1$ : $\mathrm{CX}_{3} \mathrm{C}$ chemokine receptor 1; DAMPs: Dangerassociated molecular patterns; dCas9: Dead Cas9; DMRIE: 1,2-Dimyristyloxypropyl-3-dimethyl-hydroxy ethyl ammonium bromide; DOPE: Dioleoyl phosphatidylethanolamine; DSBs: Double-strand breaks; eGFP: Enhanced
}

green fluorescent protein; EGFR: Epidermal growth factor receptor; GFP: Green fluorescent protein; GPC3: Glypican 3; GvHD: Graft-vs-host disease; hAT: hobo/Ac/Tam3; HDR: Homology-directed repair; HER-2: Human epidermal growth factor receptor 2; HSPC: Hematopoietic stem/progenitor cell; IGF-1R: Insulin-like growth factor-1 receptor; IL-2: Interleukin-2; iPSCs: Induced pluripotent stem cells; IVT: In vitro transcribed; mblL21: Membrane-bound interleukin-21; MC: Minicircle; MDA-5: Melanoma differentiation-associated protein 5; MM: Multiple myeloma; NK cells: Natural killer cells; NKG2D: Natural killer group 2D; PAMPs: Pathogen-associated molecular patterns; PB: PiggyBac; PBMCs: Peripheral blood mononuclear cells; PDCD1: Programmed cell death 1; PDX: Patient-derived xenograft; PE: Prime editor; PE: Phycoerythrin; pegRNA: Prime editing guide RNA; PRRs: Pattern recognition receptors; $\mathrm{RIG-I}$ : Retinoic acid-inducible gene I:

RNP: Ribonucleoprotein; SB: Sleeping Beauty; SCF: Stem cell factor; SLAM F7: Signaling lymphocyte activation molecule family member 7; tCTSs: Truncated Cas9 target sequences; TGFBR2: Transforming growth factor beta receptor 2; TGF $\beta$ : Transforming growth factor beta; TILs: Tumorinfiltrating lymphocytes; TIRs: Terminal inverted repeats; TLR3: Toll-like receptor 3; TME: Tumor microenvironment; TRAC: T cell receptor alpha constant; YFP: Yellow fluorescent protein

\section{Acknowledgements}

Not applicable

\section{Authors' contributions}

G.M.R. and M.W. researched the topic and co-wrote the paper. E.J.P. and B.S.M. supervised the research and edited the paper. All authors read and approved the final manuscript.

Funding

Not applicable

Availability of data and materials

Not applicable

\section{Declarations}

Ethics approval and consent to participate

Not applicable

\section{Consent for publication}

Not applicable

\section{Competing interests}

B.S.M is a founder, stockholder, and has sponsored research with Catamaran Bio, an NK therapeutics company. B.S.M and E.J.P have filed intellectual property on genome engineering of NK cells (WO2017214569A1). B.S.M is a consultant for Bio-Techne and is an inventor of hyperactive TC Buster variants. M.W. and G.M.R. have no competing interests.

\section{Author details}

${ }^{1}$ Department of Pediatrics, University of Minnesota, Minneapolis, MN 55455, USA. ${ }^{2}$ Masonic Cancer Center, University of Minnesota, Minneapolis, MN 55455, USA. ${ }^{3}$ Center for Genome Engineering, University of Minnesota, Minneapolis, MN 55455, USA. ${ }^{4}$ College of Veterinary Medicine, University of Minnesota, Saint Paul, MN 55455, USA

Received: 29 December 2020 Accepted: 21 May 2021

Published online: 16 June 2021

References

1. Gauthier J, Yakoub-Agha I. Chimeric antigen-receptor T-cell therapy for hematological malignancies and solid tumors: clinical data to date, current limitations and perspectives. Curr Res Transl Med. 2017;65(3):93-102. https:// doi.org/10.1016/j.retram.2017.08.003.

2. Fang F, Xiao W, Tian Z. NK cell-based immunotherapy for cancer. Semin Immunol. 2017:31:37-54. https://doi.org/10.1016/j.smim.2017.07.009.

3. Freund-Brown J, Chirino L, Kambayashi T. Strategies to enhance NK cell function for the treatment of tumors and infections. Crit Rev Immunol. 2018;38(2):105-30. https://doi.org/10.1615/CritRevlmmunol.2018025248. 
4. Carlsten M, Childs RW. Genetic manipulation of NK cells for cancer immunotherapy: techniques and clinical implications. Front Immunol. 2015;6:266.

5. Naeimi Kararoudi M, Tullius BP. Genetic and epigenetic modification of human primary NK cells for enhanced antitumor activity. Semin Hematol. 2020;57(4):201-12. https://doi.org/10.1053/..seminhematol.2020.11.006

6. Liu E, Marin D. Use of CAR-transduced natural killer cells in CD19-positive lymphoid tumors. N Engl J Med. 2020;382(6):545-53. https://doi.org/10.1 056/NEJMoa1910607.

7. Buchholz CJ, Friedel T, Büning $H$. Surface-engineered viral vectors for selective and cell type-specific gene delivery. Trends Biotechnol. 2015;33(12): 777-90. https://doi.org/10.1016/j.tibtech.2015.09.008.

8. Sutlu T, Nyström S. Inhibition of intracellular antiviral defense mechanisms augments lentiviral transduction of human natural killer cells: implications for gene therapy. Hum Gene Ther. 2012;23(10):1090-100. https://doi.org/1 0.1089/hum.2012.080.

9. Hu W, Wang G, Huang D, Sui M, Xu Y. Cancer immunotherapy based on natural killer cells: current progress and new opportunities. Front Immunol. 2019;10:1205. https://doi.org/10.3389/fimmu.2019.01205.

10. Lanier LL. Evolutionary struggles between NK cells and viruses. Nat Rev Immunol. 2008:8(4):259-68. https://doi.org/10.1038/nri2276.

11. Oberschmidt O, Morgan M. Development of automated separation, expansion, and quality control protocols for clinical-scale manufacturing of primary human NK cells and alpharetroviral chimeric antigen receptor engineering. Hum Gene Ther Methods. 2019;30(3):102-20. https://doi.org/1 0.1089/hgtb.2019.039.

12. Ingegnere T, Mariotti FR. Human CAR NK cells: a new non-viral method allowing high efficient transfection and strong tumor cell killing. Front Immunol. 2019;10:957. https://doi.org/10.3389/fimmu.2019.00957.

13. Deipolyi AR, Golberg A, Yarmush ML, Arellano RS, Oklu R. Irreversible electroporation: evolution of a laboratory technique in interventional oncology. Diagn Interv Radiol. 2014;20:147-54

14. Clark PR, Stopeck AT, Parker SE, Hersh EM. Cationic lipid gene transfer of an IL-2 transgene leads to activation of natural killer cells in a SCID mouse human tumor xenograft. Cell Immunol. 2000;204(2):96-104. https://doi.org/1 0.1006/cimm.2000.1699.

15. Gong JH, Maki G, Klingemann HG. Characterization of a human cell line (NK92) with phenotypical and functional characteristics of activated natural killer cells. Leukemia. 1994;8(4):652-8.

16. Zhang J, Sun R, Wei H, Zhang J, Tian Z. Characterization of stem cell factor gene-modified human natural killer cell line, NK-92 cells: implication in NK cell-based adoptive cellular immunotherapy. Oncol Rep. 2004;11:1097-106.

17. Kruschinski A, Moosmann A. Engineering antigen-specific primary human NK cells against HER-2 positive carcinomas. Proc Natl Acad Sci U S A. 2008; 105(45):17481-6. https://doi.org/10.1073/pnas.0804788105.

18. Youness RA, Rahmoon MA. Contradicting interplay between insulin-like growth factor-1 and miR-486-5p in primary NK cells and hepatoma cell lines with a contemporary inhibitory impact on HCC tumor progression. Growth Factors. 2016;34(3-4):128-40. https://doi.org/10.1080/08977194.2 016.1200571.

19. Regis S, Caliendo F. TGF- $\beta 1$ downregulates the expression of CX3CR1 by inducing miR-27a-5p in primary human NK cells. Front Immunol. 2017;8:868. https://doi.org/10.3389/fimmu.2017.00868.

20. Hargreaves BKV, Roberts SE, Derfalvi B, Boudreau JE. Highly efficient serumfree manipulation of miRNA in human NK cells without loss of viability or phenotypic alterations is accomplished with TransIT-TKO. PLoS One. 2020; 15(4):e0231664. https://doi.org/10.1371/journal.pone.0231664.

21. Wilk AJ, Weidenbacher NLB. Charge-altering releasable transporters enable phenotypic manipulation of natural killer cells for cancer immunotherapy. Blood Adv. 2020;4(17):4244-55. https://doi.org/10.1182/bloodadvances.202 0002355.

22. Pomeroy EJ, Hunzeker JT. A genetically engineered primary human natural killer cell platform for cancer immunotherapy. Mol Ther. 2020;28(1):52-63. https://doi.org/10.1016/j.ymthe.2019.10.009.

23. Schoenberg $\mathrm{K}$, Trompeter $\mathrm{H}-\mathrm{I}$, Uhrberg M. Delivery of DNA into natural killer cells for immunotherapy. Methods Mol Biol. 2008;423:165-72. https://doi. org/10.1007/978-1-59745-194-9_11.

24. Flanagan M, Gimble JM. Competitive electroporation formulation for cell therapy. Cancer Gene Ther. 2011;18(8):579-86. https:/doi.org/10.1038/cgt.2011.27.

25. Trompeter H-I, Weinhold S, Thiel C, Wernet $\mathrm{P}$, Uhrberg M. Rapid and highly efficient gene transfer into natural killer cells by nucleofection. J Immunol
Methods. 2003;274(1-2):245-56. https://doi.org/10.1016/S0022-1759(02 00431-3.

26. Carlsten $\mathrm{M}$, et al. Efficient mRNA-based genetic engineering of human NK cells with high-affinity CD16 and CCR7 augments rituximab-induced ADCC against lymphoma and targets NK cell migration toward the lymph nodeassociated chemokine CCL19. Front Immunol. 2016;7:105.

27. Boissel L, Betancur M. Comparison of mRNA and lentiviral based transfection of natural killer cells with chimeric antigen receptors recognizing lymphoid antigens. Leuk Lymphoma. 2012;53(5):958-65. https:// doi.org/10.3109/10428194.2011.634048.

28. Li L, Liu LN. Expression of chimeric antigen receptors in natural killer cells with a regulatory-compliant non-viral method. Cancer Gene Ther. 2010; 17(3):147-54. https://doi.org/10.1038/cgt.2009.61.

29. Muñoz-López M, García-Pérez JL. DNA transposons: nature and applications in genomics. Curr Genomics. 2010;11(2):115-28. https://doi.org/10.2174/13 8920210790886871.

30. Tipanee J, VandenDriessche T, Chuah MK. Transposons: moving forward from preclinical studies to clinical trials. Hum Gene Ther. 2017;28(11):1087104. https://doi.org/10.1089/hum.2017.128.

31. Kumar A. Jump around: transposons in and out of the laboratory. F1000Res. 2020:9:135. https://doi.org/10.12688/f1000research.21018.1.

32. Tipanee J, Chai YC, VandenDriessche T, Chuah MK. Preclinical and clinical advances in transposon-based gene therapy. Biosci Rep. 2017;37(6). https:// doi.org/10.1042/BSR20160614.

33. Ivics Z, Hackett PB, Plasterk RH, Izsvák Z. Molecular reconstruction of Sleeping Beauty, a Tc1-like transposon from fish, and its transposition in human cells. Cell. 1997;91(4):501-10. https://doi.org/10.1016/S0092-8674 (00)80436-5.

34. Kebriaei P, Izsvák Z, Narayanavari SA, Singh H, Ivics Z. Gene therapy with the Sleeping Beauty transposon system. Trends Genet. 2017;33(11):852-70. https://doi.org/10.1016/j.tig.2017.08.008.

35. Mátés L, Chuah MKL. Molecular evolution of a novel hyperactive Sleeping Beauty transposase enables robust stable gene transfer in vertebrates. Nat Genet. 2009;41(6):753-61. https://doi.org/10.1038/ng.343.

36. Uchida N, Hsieh MM. Optimal conditions for lentiviral transduction of engrafting human CD34+ cells. Gene Ther. 2011;18(11):1078-86. https://doi. org/10.1038/gt.2011.63.

37. Yant SR, Wu X. High-resolution genome-wide mapping of transposon integration in mammals. Mol Cell Biol. 2005;25(6):2085-94. https://doi.org/1 0.1128/MCB.25.6.2085-2094.2005.

38. Wilson MH, Coates CJ, George AL Jr. PiggyBac transposon-mediated gene transfer in human cells. Mol Ther. 2007;15(1):139-45. https://doi.org/10.1038/ sj.mt.6300028

39. Cadiñanos J, Bradley A. Generation of an inducible and optimized piggyBac transposon system. Nucleic Acids Res. 2007;35(12):e87. https://doi.org/10.1 093/nar/gkm446.

40. Yusa K, Zhou L, Li MA, Bradley A, Craig NL. A hyperactive piggyBac transposase for mammalian applications. Proc Natl Acad Sci U S A. 2011; 108(4):1531-6. https://doi.org/10.1073/pnas.1008322108.

41. Arensburger $P$, Hice $\mathrm{RH}$. Phylogenetic and functional characterization of the hAT transposon superfamily. Genetics. 2011;188(1):45-57. https://doi.org/1 0.1534 /genetics.111.126813.

42. Li X, Ewis H. A resurrected mammalian hAT transposable element and a closely related insect element are highly active in human cell culture. Proc Natl Acad Sci U S A. 2013;110(6):E478-87. https://doi.org/10.1073/pnas.1121543109.

43. Woodard LE, Li X. Comparative analysis of the recently discovered hAT transposon TcBuster in human cells. PLoS One. 2012;7(11):e42666. https:// doi.org/10.1371/journal.pone.0042666.

44. Suck G, Odendahl M. NK-92: an 'off-the-shelf therapeutic' for adoptive natural killer cell-based cancer immunotherapy. Cancer Immunol Immunother. 2016; 65(4):485-92. https://doi.org/10.1007/s00262-015-1761-x.

45. Li Y, Hermanson DL, Moriarity BS, Kaufman DS. Human iPSC-derived natural killer cells engineered with chimeric antigen receptors enhance anti-tumor activity. Cell Stem Cell. 2018;23:181-192.e5.

46. Wang J, Lupo KB, Chambers AM, Matosevic S. Purinergic targeting enhances immunotherapy of CD73+ solid tumors with piggyBac-engineered chimeric antigen receptor natural killer cells. J Immunother Cancer. 2018;6(1):136. https://doi.org/10.1186/s40425-018-0441-8.

47. Knorr DA, Ni Z. Clinical-scale derivation of natural killer cells from human pluripotent stem cells for cancer therapy. Stem Cells Transl Med. 2013:2(4): 274-83. https://doi.org/10.5966/sctm.2012-0084. 
48. Ptáčková P, Musil J. A new approach to CAR T-cell gene engineering and cultivation using piggyBac transposon in the presence of IL-4, IL-7 and IL21. Cytotherapy. 2018;20(4):507-20. https://doi.org/10.1016/j.jcyt.2017.10.001.

49. Rotiroti MC, Buracchi C. Targeting CD33 in chemoresistant AML patient-derived xenografts by CAR-CIK cells modified with an improved SB transposon system. Mol Ther. 2020;28(9):1974-86. https://doi.org/10.1016/.ymthe.2020.05.021.

50. Hu B, Zou Y. Nucleofection with plasmid DNA for CRISPR/Cas9-mediated inactivation of programmed cell death protein 1 in CD133-specific CAR T cells. Hum Gene Ther. 2019;30(4):446-58. https://doi.org/10.1089/hum.2017.234.

51. Li H, Huang Y. Antitumor activity of EGFR-specific CAR T cells against nonsmall-cell lung cancer cells in vitro and in mice. Cell Death Dis. 2018;9(2): 177. https://doi.org/10.1038/s41419-017-0238-6.

52. Xu J-Y, et al. Mesothelin-targeting chimeric antigen receptor-modified $T$ cells by piggyBac transposon system suppress the growth of bile duct carcinoma. Tumour Biol. 2017:39:1010428317695949.

53. Wang $P$, Qin W. PiggyBac-engineered T cells expressing a glypican-3specific chimeric antigen receptor show potent activities against hepatocellular carcinoma. Immunobiology. 2020;225(1):151850. https://doi. org/10.1016/j.imbio.2019.09.009

54. Magnani CF, Gaipa G. Sleeping Beauty-engineered CAR T cells achieve antileukemic activity without severe toxicities. J Clin Invest. 2020;130(11): 6021-33. https://doi.org/10.1172/JCl138473.

55. Kebriaei P, Singh H. Phase I trials using Sleeping Beauty to generate CD19specific CAR T cells. J Clin Invest. 2016;126(9):3363-76. https://doi.org/10.11 72/JCl86721.

56. A phase I/lla clinical trial to assess feasibility, safety and antitumor activity of autologous SLAMF7 CAR-T cells in multiple myeloma. https://clinicaltrials. gov/ct2/show/NCT04499339. Accessed 10 Oct 2020.

57. Thomas CE, Ehrhardt A, Kay MA. Progress and problems with the use of viral vectors for gene therapy. Nat Rev Genet. 2003;4(5):346-58. https://doi. org/10.1038/nrg1066.

58. Naeimi Kararoudi M, et al. Generation of knock-out primary and expanded human NK cells using Cas9 ribonucleoproteins. J Vis Exp. 2018. https://doi. org/10.3791/58237.

59. Liang X, Potter J. Rapid and highly efficient mammalian cell engineering via Cas9 protein transfection. J Biotechnol. 2015;208:44-53. https://doi.org/10.1 016/j.jbiotec.2015.04.024

60. Rautela J, Surgenor E, Huntington ND. Efficient genome editing of human natural killer cells by CRISPR RNP. Cold Spring Harb Lab. 2018:406934. https://doi.org/10.1101/406934.

61. Nguyen DN, Roth TL, Li PJ, Chen PA, Apathy R, Mamedov MR, et al, Polymer-stabilized Cas9 nanoparticles and modified repair templates increase genome editing efficiency. Nat Biotechnol. 2020;38(1):44-9. https:// doi.org/10.1038/s41587-019-0325-6.

62. Anzalone AV, Koblan LW, Liu DR. Genome editing with CRISPR-Cas nucleases, base editors, transposases and prime editors. Nat Biotechnol. 2020;38(7):824-44. https://doi.org/10.1038/541587-020-0561-9.

63. Komor AC, Badran AH, Liu DR. CRISPR-based technologies for the manipulation of eukaryotic genomes. Cell. 2017;169(3):559. https://doi.org/1 0.1016/j.cell.2017.04.005.

64. Komor AC, Kim YB, Packer MS, Zuris JA, Liu DR. Programmable editing of a target base in genomic DNA without double-stranded DNA cleavage. Nature. 2016;533(7603):420-4. https://doi.org/10.1038/nature17946.

65. Gaudelli NM, Komor AC, Rees HA, Packer MS, Badran AH, Bryson Dl, et al. Programmable base editing of $A \cdot T$ to $G \cdot C$ in genomic DNA without DNA cleavage. Nature. 2017;551(7681):464-71. https://doi.org/10.1038/nature24644.

66. Webber BR, et al. Highly efficient multiplex human T cell engineering without double-strand breaks using Cas9 base editors. Nat Commun. 2019; 10:5222.

67. Shi X, Zhang D, Li F, Zhang Z, Wang S, Xuan Y, et al. Targeting glycosylation of PD-1 to enhance CAR-T cell cytotoxicity. J Hematol Oncol. 2019;12, 127(1). https://doi.org/10.1186/s13045-019-0831-5.

68. Anzalone AV, Randolph PB, Davis JR, Sousa AA, Koblan LW, Levy JM, et al. Searchand-replace genome editing without double-strand breaks or donor DNA. Nature. 2019;576(7785):149-57. https://doi.org/10.1038/s41586-019-1711-4.

\section{Publisher's Note}

Springer Nature remains neutral with regard to jurisdictional claims in published maps and institutional affiliations. 